\title{
Alterations des fonctions de reproduction chez les souris males deficientes en recepteur aux estrogenes
}

\author{
K.S KORACH, J.LINDZEY \\ Receptor Biology Section, Laboratory of Reproductive and Developmental Toxicology, National Institute of \\ Environmental Health Science, National Institute of Health, Research Triangle Park, USA.
}

Traduction : par S. Carreau

\section{RESUME}

La délétion du récepteur aux estrogènes (estrogen receptor knock out i.e ERKO) chez la souris mâle conduit à la stérilité suite à une déficience de la gamétogenèse associée à des altérations du comportement sexuel. En fonction de l'âge, le développement testiculaire est de plus en plus anormal et les cellules germinales dégénèrent. Les taux circulants de gonadotropines et de testostérone ne sont pas significativement plus élevés chez les mâles ERKO ce qui suggère que les androgènes testiculaires exercent correctement leur contrôle sur les secrétions de gonadotropines même en absence d'aromatisation et d'activation du récepteur aux estrogènes (ER). La stérilité des souris mâles ERKO n'est pas la conséquence d'une modification du rôle des androgènes puique la morphologie du tractus génital n'est pas altérée. Le comportement de monte des mâles est normal alors que l'accouplement et l'éjaculation sont sévèrement compromis.

Mots clés: ERKO, Reproduction, Récepteur estrogène, Souris.

\section{INTRODUCTION}

Depuis toujours on attribue aux androgènes testiculaires un rôle régulateur dans le développement mâle et les fonctions de reproduction. L'action des androgènes peut être modulée directement par la liaison de la testostérone $(\mathrm{T})$ ou de la dihydrotestostérone (DHT) au récepteur des androgènes (AR) ou indirectement, via leur transformation en estrogènes et l'activation ultérieure de ER. Cette dernière possibilité rend compte de nombreux effets activateurs et «organisateurs» des androgènes au niveau cérébral et du comportement des mâles [16]. Alors que les effets cérébraux de l'estradiol (E2) sur le comportement du mâle sont bien acceptés, le rôle de l' $\mathrm{E} 2$ endogène dans les autres domaines de la physiologie mâle a été peu abordé. Cependant, le développement récent du modèle ERKO chez la souris a permis d'apporter des renseignements utiles quant au rôle régulateur de l'E2 dans le développement et les fonctions de reproduction chez la souris adulte tant au niveau central (nerveux) que périphérique (gonades et glandes annexes).

L'expression du gène codant pour le récepteur aux estrogènes a été interrompue par l'insertion d'un marqueur spécifique du gène de la néomycine dans le second exon du gène ER de souris [9-11]. Les animaux hétérozygotes sont fertiles et semblent normaux en dépit d'une expression $50 \%$ plus faible du taux de ER. Le 
croisement des hétérozygotes donne des animaux viables avec des génotypes qui suivent les lois de Mendel [11]. Les mâles homozygotes pour la délétion du gène $\mathrm{ER}$ sont stériles suite à des anomalies testiculaires et des altérations du système nerveux affectant le comportement sexuel.

\section{LES TESTICULES}

Chez les mâles ERKO le dysfonctionnement testiculaire est progressif car les testicules sont normaux à 10 jours d'âge puis une dilatation de la lumière des tubes séminifères apparaît au 20ème jour de la vie [3]. Chez les animaux âgés de 10 semaines, les testicules présentent une atrophie caractérisée par des lumières de tubes séminifères dilatées, une taille des cellules épithéliales réduite, une diminution de la spermatogenèse et une réduction du nombre des spermatozoïdes épididymaires (nombre égal à $10 \%$ du type sauvage). Ces changements progressifs sont orientés de la partie caudale vers la partie apicale testiculaires. Il est important de souligner qu'à un âge où la spermatogenèse est encore présente ( 8 semaines), le sperme des souris ERKO présente in vitro une diminution de la capacitation [3]. Chez les mâles adultes ERKO, la morphologie épididymaire est normale alors que des modifications testiculaires sont observées. Le faible nombre de spermatozoïdes enregistré chez les ERKO est conflictuel par rapport aux résultats publiés chez un homme présentant un syndrome de résistance aux estrogènes où le nombre de spermatozoïdes se trouve dans la limite inférieure de la normale [19]. Bien que la finalité de ces données portant sur un seul patient soit discutable, il serait intéressant de connaître le devenir de cette numération spermatique au cours du temps afin de voir si une décroissance s'installe comme cela est observé dans le modèle ERKO.

Les causes de l'altération de la spermatogenèse sont intéressantes à élucider. Puisque cette rupture s'opère en présence de taux de FSH circulante normaux (voir plus loin), il est concevable que des effets locaux au niveau testiculaire plutôt que des modifications systémiques des hormones soient mis en jeu. Le premier signe de dilatation des tubes séminifêres apparaît en même temps que la formation des jonctions serrées Sertoli-Sertoli et la mise en place de la barrière hémato-testiculaire. Cela amène donc à évoquer des anomalies dans les interactions cellule-cellule et, par conséquent, des altérations au niveau du fluide intratesticulaire et de la pression [3]. De fait, des différences dans les molécules d'adhésion impliquées dans la formation des jonctions serrées peuvent être envisagées puisque d'une part, des travaux ont montrés que la régulation des cadhérines est estrogénodépendantes [12] et d'autre part, des données préliminaires [Blaschuk, communication personnelle] indiquent que la $\mathrm{N}$-cadhérine est plus faiblement exprimée dans les testicules des souris ERKO adultes.

\section{LES HORMONES}

Etant donné que l'E2 et la testostérone contrôlent par feedback les productions de GnRH et de gonadotropines [4, 6], les souris ERKO devraient avoir des taux plus élevés de gonadotropines. De fait, chez l'homme présentant un syndrome de résistance aux estrogènes, les taux sériques de LH et FSH sont environ 2 fois plus élevés par rapport à la valeur supérieure de la normale [19]. Cependant chez les souris mâles ERKO, les concentrations de LH et de FSH ne sont que légèrement supérieures (non significatif) par rapport à celles des mâles contrôles [3]. De plus, les expériences de castration et de supplémentations hormonales ont démontré que les traitements à l'estradiol et la testostérone des castrats sauvages sont efficaces pour restaurer à des niveaux normaux (comparables à ceux de la souris intacte) le contenu hypothalamique en GnRH et le taux circulant de LH. Par contre, le traitement par l'estradiol des souris ERKO castrées s'est avéré inefficace tant pour le contenu en GnRH que pour la LH circulante alors que la testostérone et la DHT assurent un retour à la normale des valeurs de $\mathrm{GnRH}$ et diminuent la LH circulante (résultats non publiés). Ces données suggèrent que les androgènes testiculaires sont capables d'agir via les $\mathrm{AR}$ pour réguler le GnRH et la $\mathrm{LH}$ et donc, sont en accord avec les observations 
faites chez l'homme montrant des taux élevés de LH dans les syndromes d'insensibilité aux androgènes [5]. De la même manière, nos résultats sont conformes à ceux observés après traitement par la $\mathrm{T}$ ou la DHT chez des rats mâles où les taux circulants de $\mathrm{LH}$ sont abaissés tout comme les contenus hypophysaires en ARNm codant pour la LHB [4]. Puisque les taux de FSH sont normaux chez les ERKO cela implique que les androgènes ou les inhibines sont efficaces pour réguler la synthèse et la secretion de la FSH. Nous sommes actuellement en train de préciser le rôle des androgènes et des inhibines/activines dans le contrôle de la FSH chez les souris mâles ERKO.

La stéroïdogenèse testiculaire est normale excepté que chez les souris ERKO le taux de testostérone sérique soit 1,8 fois plus élevé [3]. Puisque les taux de LH circulants sont normaux, cela suggére que les valeurs élevées de testostérone sont la conséquence de l'absence de régulation testiculaire (via les récepteurs d'estrogènes) de la production de testostérone. Cette régulation ultra-courte peut se faire à travers le contrôle du nombre de cellules de Leydig pendant le développement, mais aussi par des modifications au niveau des récepteurs LH et donc de la réponse leydigienne. Des résultats préliminaires sont en faveur d'une augmentation des ARN messagers des récepteurs LH et FSH (4-5 et 2-3 fois, respectivement) dans les testicules des souris ERKO (non publiés); ainsi il est donc possible que les testicules de ces animaux soient plus sensibles à la LH. Des études en fonction du développement ont démontré que les traitements in utéro ou néonataux par des estrogènes modifient la taille et la capacité de certains organes androgéno-sensibles à répondre aux androgènes $[1,15,17]$. Certains de ces effets ont été reliés à la diminution du taux d'AR et de $5 \alpha$ réductase; cependant, il reste à déterminer si l'absence de ER modifie la réponse androgénique testiculaire. Chez les souris mâles ERKO et sauvages, nous sommes en train d'évaluer la capacité stéroïdogène des testicules en réponse aux gonadotropines, les niveaux de récepteurs LH ainsi que les quantités d'enzymes stéroïdiennes.

\section{GLANDES ANNEXES DU TRACTUS GENITAL MALE}

Bien que les mâles adultes ERKO soient stériles et présentent des testicules plus petits avec une spermatogenèse altérée, il n'y a pas de différence dans la taille, le poids ou la fonction des glandes annexes composant le tractus génital tant en ce qui concerne les vésicules séminales que la prostate [3]. Tous les organes dérivés du canal de Wolff sont normaux et il ne reste aucun vestige des canaux de Müller. Le développement normal de ces glandes annexes (vésicules séminales, prostate et épididyme) suggère que leur réponse androgénique est normale même si des altérations de la fonction épididymaire sont possibles puisque la capacitation des spermatozoïdes des souris ERKO est compromise.

Alors que la morphologie apparente des glandes annexes apparaît normale, le sac crémastérique est plus petit et le muscle crémaster est épaissi à la fois chez les hétérozygotes et les mâles ERKO [2]. Néanmoins, les testicules des mâles quel que soit le génotype sont réduits. Il est intéressant de noter que chez les mâles hétérozygotes et ERKO les testicules sont remontés dans le sac inguinal ou l'abdomen. Ces résultats suggèrent que soit les taux faibles ou l'absence de ER, pourraient influencer très finement la regression du gubernaculum; de plus il se pourrait que la descente testiculaire soit altérée en raison des effets sur le nerf genitofémoral et / ou la production du peptide apparenté à la calcitonine[8]. Là encore, il reste à établir si ces effets sont dûs à l'action directe des estrogènes dans ces tissus cibles ou bien à un effet indirect via la réponse androgénique.

\section{LE COMPORTEMENT MALE}

L'aromatisation de la testostérone est essentielle pour la masculinisation des noyaux hypothalamiques impliqués dans le contrôle du comportment sexuel et l'agressivité [16]. Les études initiales réalisées sur le comportement des mâles ERKO ont révélés que les animaux présentaient des comportements de monte normaux tout comme le temps de latence pour la première monte. Cependant l'accou- 
plement et l'éjaculation sont sévèrement altérés [13]. Des études complémentaires sur le comportement de reproduction ont montré que les pelotes vaginales n'ont été retrouvées que dans 2 cas sur 15 , mettant en évidence un sérieux problème d'accouplement [3]. Ces données confirment que les androgènes via les $\mathrm{AR}$, sont responsables des comportements de monte alors que l'accouplement et l'éjaculation sont régulés et / ou activés par les estrogènes via les ER chez la souris mâle. Il serait intéressant de savoir si ces déficits sexuels sont dûs à des modifications centrales (noyau préoptique / hypothalamique antérieur ou noyau spinal) ou périphériques (muscles impliqués dans les réflexes péniens).

Dans les tests d'agressivité vis à vis d'un animal étranger, les souris mâles ERKO présentent des taux d'attaques plus faibles; de plus, ils ont des comportements agressifs plus proches de ceux des femelles sauvages [13]. Ainsi ces obesrvations démontrent clairement que les mécanismes mis en jeu dans le déclenchement et l'intensité du comportement agressif sont estrogéno-dépendants.

Les altérations du comportement chez les mâles ERKO suggérent que ces animaux seraient un bon modèle pour analyser les effets des estrogènes dans le développement du cerveau et la mise en place des comportements sexuels spécifiques. Des travaux sont en cours pour examiner ces différences dans la mise en place estrogéno-dépendante du système nerveux afin de comprendre l'origine des pathologies comportementales des mâles ERKO. Toutefois à l'heure actuelle le seul changement important observé chez ces mâles ERKO [18] est la féminisation des noyaux de l'aire périventriculaire antéroventrale $(\mathrm{AVPv})$ . L'AVPv a été impliquée dans la régulation de la secrétion des gonadotropines et est reliée au sexe, car les femelles possèdent plus de cellules et de fibres exprimant la tyrosine hydroxylase (TH), une enzyme impliquée dans la synthèse de dopamine. Les noyaux de l'aire AVPv des mâles ERKO ressemblent à ceux des femelles sauvages en ce qui concerne le nombre de cellules $\mathrm{TH}$ positives [18].

\section{CONCLUSIONS}

Le modèle souris ERKO a permis d'apporter des éclaircissements quant au rôle des estrogènes dans la physiologie de la reproduction chez le mâle. Cependant, il est difficile de déterminer si un phénotype donné est la conséquence d'une absence locale d'effet des ER ou de modifications dans les taux circulants d'hormones ou de facteurs de croissance. De plus, dans le modèle ERKO, il est actuellement impossible de distinguer les effets sur le développement de ceux sur l'activation pour ce qui est de la délétion de ER. En conséquence il serait important de développer de nouvelles lignées de souris chez lesquelles l'expression de ER est reciblée dans des organes spécifiques de la lignée ERKO. En somme, notre approche vise le développement de modèles knockout tissu-spécifiques et inductibles afin d'avoir des outils pour définir avec précision les sites exacts et à quel moment les estrogènes et les ER interviennent dans la régulation du développement reproducteur et la physiologie chez le mâle.

\section{REFERENCES}

1. BECKMAN WC., NEWBOLD RR., TENG CT., MCLACHLAN JA.: Molecular feminization of mouse seminal vesicles by prenatal exposure to diethylstilbestrol: altered expression of messenger RNA. J.Urol., 1994; 151: 1370-1378.

2. DONALDSON KM., TONG SYC., WASHBURN TF., et al. : Morphometric study of the gubernaculum in male estrogen receptor mutant mice. J.Androl., 1996; 17 : 91-95.

3. EDDY EM., WASHBURN TF., BUNCH DO., et al. : Targeted disruption of the estrogen receptor gene in male mice causes alteration of spermatogenesis and infertility. Endocrinology, 1996; $137:$ 4796-4805.

4. GHARIB S., WIERMAN M., SHUPNIK M., CHIN W. : Molecular biology of the pituitary gonadotropins. Endocr.Rev., 1990; 11: 177-199.

5. GRIFFIN JE., WILSON JD. : The androgen resistance syndromes: $5 \alpha$-reductase deficiency, testicular feminization and related disorders. In: SCRIVER CR., BEAUDET AL., SLY WS., VALLE D., eds. The Metabolic Basis of Inherited Disease, 6th ed. New York, McGraw-Hill, 1989; pp : 1919-1944.

6. HAISENLEDER DJ., DALKIN AC., MARSHALL JC.: Regulation of gonadotropin gene expression. In : KNOBIL E., NEILL JD., eds. The Physiology of Reproduction, 2nd ed. New York, Raven Press, Ltd, 
1994; pp : 1793-1813.

7. HALES D., PAYNE AH. : Glucocorticoid-mediated repression of P450scc mRNA and de novo synthesis in cultured Leydig cells. Endocrinology, 1989; 124 : 20992104.

8. HUTSON JM., TERADA M., ZHOU BY., WILLIAMS MPL. : Normal testicular descent and the etiology of cryptorchidism. In: BECK F., ed. Advances in Anatomy, Embryology and Cell Biology. Berlin, SpringerVerlag, 1996; pp: 1-56.

9. KORACH KS. : Insights from the study of animals lacking functional estrogen receptor. Science, 1994; 266 : 1524-1527.

10.KORACH KS., COUSE JF., CURTIS SW., et al. : Estrogen receptor gene disruption : molecular characterization and experimental and clinical phenotypes. Rec. Prog. Horm. Res., 1996; 51 : 159-188.

11.LUBAHN DB., MOYER JS., GOLDING TS., COUSE JF., KORACH KS., SMITH ESO. : Alteration of reproductive function but not prenatal sexual development after insertional disruption of the mouse estrogen receptor gene. Proc. Natl. Acad. Sci. USA, 1993; 90 : 11162-11166.

12. MCCALMAN CD., FAROOKHI R., BLASCHUK OW. : Estradiol regulates $\mathrm{N}$-cadherin mRNA levels in mouse ovary. Dev.Genet., 1995; $16: 20-24$.

13. OGAWA S., LUBAHN DB., KORACH KS., PFAFF DW. : Behavioral characteristics of transgenic estrogen receptor knock out male mice: sexual, aggressive and open-field behaviors. Proc. 77th Annual Meeting Endocrine Society Washington DC, 1995; p: 133.

14. PAYNE AH., YOUNGBOLLD G. : Regulation of expression of steroidogenic enzymes in Leydig cells. Biol. Reprod;, 1995; 52 : 217-225.

15. PYLKKANEN L., SANTTI R., NEWBOLD RR., MCLACHLAN JA. : Regional differences in the prostate of the neonatally estrogenized mouse. Prostate, $1991 ; 18: 117-129$.

16. SACHS BD., MEISEL RL. : The physiology of male sexual behavior. In : KNOBIL E, NEILL JD, eds. The Physiology of Reproduction, 2nd ed. New York, Raven Press Ltd., 1994; 2 : 3-106.

17. SANTTI R., NEWBOLD RR., MCLACHLAN JA. : Androgen metabolism in control and neonatally estrogenized male mice. Reprod. Toxicol., 1991; 5 : 149-155.

18. SIMMERLY RB., KORACH KS., LUBAHN DB., ZEE M. : Disruption of the estrogen receptor gene interferes with sexual differentiation of dopamine neurons in the peoptic region of the mouse. Society of Neuroscience, 1996; $22: 561$ (abstract).

19. SMITH EP., BOYD J., FRANK G., et al. : Estrogen resistance caused by a mutation in the estrogenreceptor gene in a man. N. Engl. J. Med., 1994; 331 : 1056-1061.
20. VALE W., BILEZIKJIAN L., RIVIER C. : Reproductive and other roles of inhibins and activins. In : KNOBIL E, NEILL JD, eds. The Physiology of Reproduction, 2nd ed. New York, Raven Press Ltd., 1994;pp : 1861-1878.

\section{ABSTRACT}

Alterations in male reproduction in estrogens receptor knock out mice

K.KORACH, J. LINDZEY

In male mice, ER disruption causes infertility due to deficiencies in both gametogenesis and sexual behavior. An age dependent development of testicular dysmorphogenesis results in a progressive loss of germ cells. Circulating gonadotropins and testosterone are not appreciably higher in ERKO males, indicating that testicular androgens can effectively exert feedback on gonadotropins in the absence of aromatization and activation of ER. Infertility in male mice ERKOs does not appear to be a result of compromised androgen hormone action since the morphology of male reproductive tract organs is largely unaffected. Mounting behavior of ERKO males is normal whereas the ability to intromit and ejaculate is severely compromised.

Key words: ERKO, Reproduction, Estrogen Receptor, Mice. 